David KOLLOSCHE, Potsdam

\title{
Gesellschaftliche Funktionen des Mathematikunterrichts
}

Während die normative Frage, was Mathematikunterricht leisten soll, ein traditionelles Arbeitsfeld der Mathematikdidaktik ist, gibt es auf die deskriptive Frage, was gegenwärtiger Mathematikunterricht an deutschen Sekundarschulen tatsächlich leistet, kaum Antworten. Dabei hat gerade eine soziologische Analyse der gesellschaftlichen Funktionen des Mathematikunterrichts das Potential, diesen in seinen Möglichkeiten und Grenzen zu erkennen, auf diese in Forschung und Praxis zu reagieren und so Zielkonflikte zu vermeiden, welche dem Erfolg mathematikdidaktischer Interventionen im Wege stehen können.

Helmut Fends legte mit einer Soziologie der Schule (1974) eine Typologie gesellschaftlicher Funktionen von Schule vor. Demnach hat Schule nicht nur die Aufgabe einer Qualifikation der Heranwachsenden, sondern muss die bestehenden Formen gesellschaftlichen Denkens und Tuns legitimieren, die Schüler in diese Formen integrieren und sie schließlich an geeigneten Maßstäben bewerten. Da Studien wie die von Jean Lave (1988) zeigen, dass außerschulisch verwendete Mathematik meist nicht in der Schule, sondern im Verwendungskontext gelernt wurde, und da die Inhalte des Mathematikunterrichts ab der achten Klasse in der Regel nur von wenigen Menschen außerschulisch verwendet werden (vgl. Heymann 1996, S. 153), ist der Qualifikationsfunktion des Mathematikunterricht zumindest skeptisch zu begegnen und zu vermuten, dass andere gesellschaftliche Funktionen wenigstens ebenso prägend sind.

Fraglich ist also, welches gesellschaftliches Denken und Tun im Mathematikunterricht legitimiert und kultiviert wird. Erste Anhaltspunkte dazu finden sich in kritischer Forschung zum Mathematikunterricht. So heißt es bei Christine Keitel (1979) und Philipp Ullmann (2008), dass der Mathematikunterricht die Mathematik als gesellschaftliches Machtmittel etabliere, indem er vermittle, dass alle weltlichen Probleme mathematisierbar und dann eindeutig lösbar seien. Roland Fischer (2001) und Ole Skovsmose (2005, S. 11f.) verweisen auf unterschiedliche Aspekte der Verwandtschaft von Mathematik und Bürokratie, so dass wenigstens die Frage im Raum steht, inwiefern der Mathematikunterricht bürokratisches Denken vorbereitet. Schließlich sehen soziolinguistisch inspirierte Beiträge (Dowling 1998) im Mathematikunterricht allgemein eine Institution zur Reproduktion und Inthronisierung elaborierter Formen des Denkens und Sprechens.

In J. Roth \& J. Ames (Hrsg.), Beiträge zum Mathematikunterricht 2014 (S. 635-638). Münster: WTM-Verlag 
Problematisch ist an diesen Beiträgen jedoch zweierlei: Zum einen sind sie soziologisch nicht konsistent, sondern verwenden oft unterschiedliche, sich teilweise widersprechende soziologische Ansätze in willkürlicher Zusammenstellung, was die Interpretation der vorgebrachten Thesen erheblich erschwert. Nötig ist stattdessen eine Soziologie des Mathematikunterrichts, die die Funktionen von Mathematikunterricht sowohl auf der gesellschaftlichen Makroebene, als auch auf der individuellen Mikroebene zu beschreiben imstande ist. $\mathrm{Zu}$ diesem Zweck lässt sich die Soziologie von Michel Foucault ausarbeiten (vgl. Kollosche 2012). Zum anderen betrachten diese Beiträge die Mathematik in ihrer reinen Form meist als Phänomen jenseits kultureller Einflüsse, welches erst durch die Form seiner Anwendung oder Lehre politisch wird. Eine solche Sicht versperrt jedoch den Blick auf kulturelle Dimensionen der Mathematik an sich, weshalb diese in den genannten Beiträgen selten zum Untersuchungsgegenstand werden. Um diese Lücke zu schließen, wurden in genealogischen Studien zur Logik (vgl. Kollosche 2013a) und zum Rechnen (vgl. Kollosche 2013b) zwei zentrale Aspekte von Mathematik unter die soziologische Lupe genommen.

Die gemeinsame Betrachtung der gesellschaftlichen Makro- und Mikroebene gelingt Foucault (1994) durch seinen Begriff der Führungstechniken, deren Verfügung das ist, was Foucault Macht nennt. Er unterscheidet zwischen Techniken zur Führung anderer und Techniken zur Führung des Selbst und interessiert sich besonders für sogenannte Disziplinartechniken, d. h. Techniken zur Führung anderer durch deren Selbstführungstechniken. Eine schlechte Note für Fehler beim Konstruieren im Geometrieunterricht dient als Technik zur Führung anderer (der Schüler) durch den Lehrer; doch um wie vom Lehrer erwünscht konstruieren zu können, muss der Schüler Selbstführungstechniken ausbilden - ein Prozess, den Foucault Askese nennt, den der Lehrer nicht steuern kann, der je individuell verläuft und der schließlich zur Internalisierung ursprünglich externer Forderungen durch das Individuum führt. In dieser Internalisierung sieht Foucault die Effizient der in der Neuzeit populären Disziplinartechniken: sie müssen sich nicht bestätig aufdrängen, sondern wirken alsbald aus dem Einzelnen heraus, wie womöglich aus dem Schüler, der nun auch von anderen saubere Konstruktionen erwartet und auch außerhalb des Mathematikunterrichts gewissenhaftere Zeichnungen anstellt.

Da der Mathematikunterricht die Schüler Situationen aussetzt, die nur mathematisch zu bewältigen sind, in keinem Fall jedoch die genaue Technik zur Lösung vorgeben wird, lässt er sich verstehen als Disziplinarinstitution, die bestimmte Formen des gesellschaftlichen Denkens legitimiert und kultiviert, den Schülern also eine entsprechende Askese abverlangt. 
Indem das Verständnis mathematischer Konzepte mit dem logischen eine sehr spezielle Form des Denkens verlangt, ist die Beschäftigung mit Mathematik eine Disziplinartechnik, welche eine Askese im logischen Denken verlangt. Damit wird im Mathematikunterricht eine Form des Denkens und Sprechens legitimiert, welche religiöse, epistemologische und politische Dimensionen hat. Einerseits bietet die Logik Beruhigung durch Wahrheitsglaube, eine Ordnung des Denkens und eine Technik zur Konsensfindung jenseits physischer Gewalt; andererseits führt sie zu einem statischen Weltbild, einer Reduktion des Denkbaren und zur Etablierung neuer Unterdrückung (vgl. Kollosche 2013a).

Neben der Logik und anderen ist auch das Rechnen ein zentraler Aspekt der Mathematik, insbesondere im Mathematikunterricht. Für das Rechnen lässt sich an Hand der Genese des Rechnens (Krämer 1988) und Max Webers Theorie der Bürokratie (1972, S. 551ff.) nicht nur aufzeigen, dass Entwicklungsschübe in der Formalisierung des Rechens meist zeitlich und örtlich mit der Etablierung bürokratischer Verwaltungsstrukturen einhergehen, sondern auch, dass beide sehr ähnlicher Selbstführungstechniken bedürfen. Sowohl das Rechnen als auch die Bürokratie reduzieren reale Probleme auf Modelle bzw. Fälle, also auf eine regelhaft handhabbare Vereinfachungen, die im Folgenden an Hand fremdbestimmter Regeln und unter Ignoranz aller persönlicher Empfindungen zu bearbeiten sind. Dazu bedarf es seitens des Bearbeiters nicht nur der Fähigkeit zur reduktionistischen Wahrnehmung von Wirklichkeit in Fällen, zu ihrer rein regelhaften Bearbeitung und zum Ausschalten aller persönlichen Einflüsse, sondern auch der Bereitschaft dazu. Diese Fähigkeit und Bereitschaft im Heranwachsenden auszubilden und so jene unpersönliche Verwaltung zu ermöglichen, welche in der Neuzeit gerecht genannt wird, mag eine Funktion des Rechenunterrichts sein (vgl. Kollosche 2013b).

In beiden Fällen sind die Disziplinartechniken, die eine Askese zum logischen bzw. bürokratischen Denken anstoßen, so gestaltet, dass sie dem Schüler idealtypisch nur die Wege der Komplizenschaft oder Askese offenhalten. Entweder ist der Schüler fähig und willig, logisch bzw. bürokratisch zu denken und zu handeln, und ermöglicht sich dadurch Erfolgserlebnisse im Mathematikunterricht sowie gute Bewertungen, oder er nicht dazu nicht fähig oder nicht willig, in welchem Fall er sich durch andauernde Misserfolgserlebnisse gepeinigt vom Mathematikunterricht und der Mathematik abwenden wird. Auf diese Weise produziert der Mathematikunterricht auf der einen Seite Komplizen seiner Machttechniken und sorgt auf der anderen Seite dafür, dass Zweifler nicht aufbegehren, sondern verstummen und das Feld den Willigen überlassen. Eine solche Wirkungsweise hatte 
Skovsmose (2005, S. 11f.) freilich schon allgemein hinter dem Mathematikunterricht vermutet. In der Tat können erst durch dieses Verhindern eines Dissenses bezüglich der Legitimität des logischen Denkens und des Rechnens beide zu gesellschaftstragenden Führungstechniken avancieren.

Abgesehen vom moralischen Dilemma, welches durch den Konflikt zwischen gesellschaftstragender Sozialisation einerseits und individuellem Emanzipationsbestreben andererseits entsteht, führen die obigen Überlegungen zur Einsicht, dass eine soziologische Betrachtung des Mathematikunterrichts mathematikdidaktisch durchaus relevante Erkenntnisse bereithält, das Forschungsgebiet jedoch noch in seinen Kinderschuhen steckt. Es bleibt zumindest die Hoffnung, dass eine weitere soziologische Untersuchung des Mathematikunterrichts dazu beträgt, seine gesellschaftlichen Funktionen besser zu verstehen und mathematikdidaktisch effizienter auf diese reagieren zu können.

\section{Literatur}

Dowling, Paul (1998) The Sociology of Mathematics Education. Falmer: London.

Fend, Helmut (1974) Gesellschaftliche Bedingungen schulischer Sozialisation. Beltz: Weinheim.

Fischer, Roland (2001) »Mathematik und Bürokratie« in Lengnink et. al. (Hg.) Mathematik und Mensch. Sichtweisen der allgemeinen Mathematik. Allg. Wiss.: Mühltal. S. 53-64.

Foucault, Michel (1994) »Wie wird Macht ausgeübt?« in Dreyfus \& Rabinow (Hg.) Michel Foucault. Jenseits von Strukturalismus und Hermeneutik. Beltz: Weinheim. S. 251-261.

Heymann, Hans Werner (1996) Allgemeinbildung und Mathematik. Beltz: Weinheim.

Keitel, Christine (1979) »Sachrechnen« in Dieter Volk (Hg.) Kritische Stichwörter zum Mathematikunterricht. Wilhelm Fink: München. S. 249-264.

Kollosche, David (2012) »Foucault und Mathematikdidaktik - eine fruchtbare Mischung?« in Ludwig \& Kleine (Hg.): Beiträge zum Mathematikunterricht 2012. VTM: Münster.

Kollosche, David (2013) »Logik, Gesellschaft, Mathematik « in Martin Rathgeb (Hg.) Mathematik im Prozess. Springer: Wiesbaden. S. 29-40.

Kollosche, David (2013) »Bürokratie und Rechnen im Mathematikunterricht" in Greefrath et. al. (Hg.): Beiträge zum Mathematikunterricht 2013. VTM: Münster.

Krämer, Sybille (1988) Symbolische Maschinen. WBG: Darmstadt.

Lave, Jean (1988) Cognition in Practice. Mind, Mathematics and Culture in Everyday Life. Cambridge University Press: Cambridge.

Skovsmose, Ole (2005) Travelling Through Education. Uncertainty, Mathematics, Responsibility. Sense: Rotterdam.

Ullmann, Philipp (2008) Mathematik, Moderne, Ideologie. Eine kritische Studie zur Legitimität und Praxis der modernen Mathematik. UVK: Konstanz.

Weber, Max (1922) Wirtschaft und Gesellschaft. Grundriss der verstehenden Soziologie. Erw. Ausgabe von 1972. Hg. von Johannes Winckelmann. Mohr: Tübingen. 